\title{
Group 4 Metal Complexes of Nitrogen-bridged Dialkoxide Ligands: Synthesis, Structure and Polymerization Activity Studies
}

Laurent Lavanant, ${ }^{\dagger}$ Loic Toupet, ${ }^{\ddagger}$ Christian W. Lehmann, ${ }^{\$}$ and Jean-François Carpentier ${ }^{\dagger, *}$

Organométalliques et Catalyse, UMR 6509 CNRS-Université de Rennes 1, Institut de Chimie de Rennes, 35042 Rennes Cedex, France

Groupe Matière Condensée et Matériaux, Cristallochimie, UMR 6626 CNRS-Université de Rennes 1, 35042 Rennes Cedex, France

Max-Planck-Institut für Kohlenforschung, Chemical Crystallography, Postfach 101353, 45466 Mülheim/Ruhr, Germany

I.1. Variable temperature ${ }^{1} \mathrm{H}$ NMR spectra of $\left\{\mathrm{ONO}^{\mathrm{tol}}\right\} \mathrm{TiCl}_{2}$ (14)

I.2. Variable temperature ${ }^{1} \mathrm{H}$ NMR spectra of $\left\{\mathrm{ONO}^{\mathrm{Me}}\right\}_{2} \mathrm{Ti}$ (5)

II. Representative GPC and DSC analyses of PE samples

† Organométalliques et Catalyse

¥ Groupe Matière Condensée et Matériaux

\$ Max-Planck-Institut

* Corresponding author: Fax: (+33)(0)223-236-939; E-mail: jean-francois.carpentier@univ$\underline{\text { rennes1.fr }}$ 
I.1. Variable temperature ${ }^{1} \mathrm{H}$ NMR spectra of $\left\{\mathrm{ONO}^{\text {tol }}\right\} \mathrm{TiCl}_{2}$ (14) (toluene- $d_{8}, 300 \mathrm{MHz}$ ) showing the $\mathrm{NCH}_{2} \mathrm{Ph}(\delta 4.05$ at $298 \mathrm{~K})$ and $\mathrm{NCH}_{2} \mathrm{CMe}_{2}(\delta$ 3.91-3.77) resonances. The vertical expansion is different in each case for clarity.

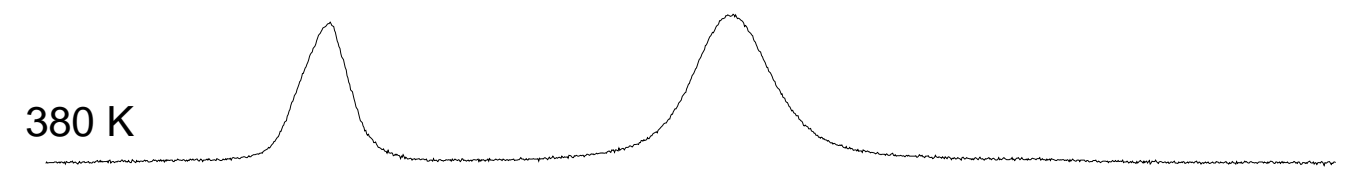

$355 \mathrm{~K}$

$340 \mathrm{~K}$

298 K 
I.2. Variable temperature ${ }^{1} \mathrm{H}$ NMR spectra of $\left\{\mathrm{ONO}^{\mathrm{Me}}\right\}_{2} \mathrm{Ti}$ (5) (toluene- $d_{8}, 300 \mathrm{MHz}$ ) showing the $\mathrm{NCH}_{2} \mathrm{Ph}(\delta 4.69$ and 4.12 at $193 \mathrm{~K})$ and $\mathrm{NCH}_{2} \mathrm{CMe}_{2}(\delta 3.08-2.35)$ resonances. The vertical expansion is different in each case for clarity..

$298 \mathrm{~K}$

$250 \mathrm{~K}$

$223 \mathrm{~K}$

$193 \mathrm{~K}$

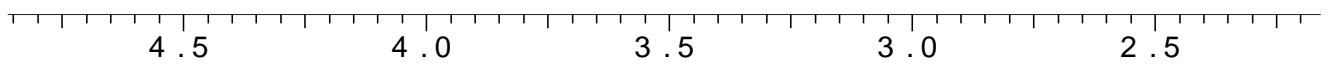


II.1. GPC of PE sample, Table 8, entry 5

\section{Polymer Laboratories}

PL Caliber GPC Software

Unknown LOLO214.HOO

$>P E$

Concentration

Injection Volume

Solvent

Column Set

Method 1

Comments :

Calibration Using : Narrow Standards

Calibration Limits :

Flow Rate Marker :

Broad Peak Start :

14.03

to 23.47

found at: Not Found

End: 19.33

14.3

\section{1:02 Tue Jun 072005}

Acquired : $\quad$ 10:41 Tue Jun 072005

Operator Richard Jordan

Detector

Temperature :

Flow Rate : 1.000

Standards :

Curve Used : $\quad 3$ rd Order Polynomial

Last Calibrated : Tue Jun 07 10:53:26 2005

Mins

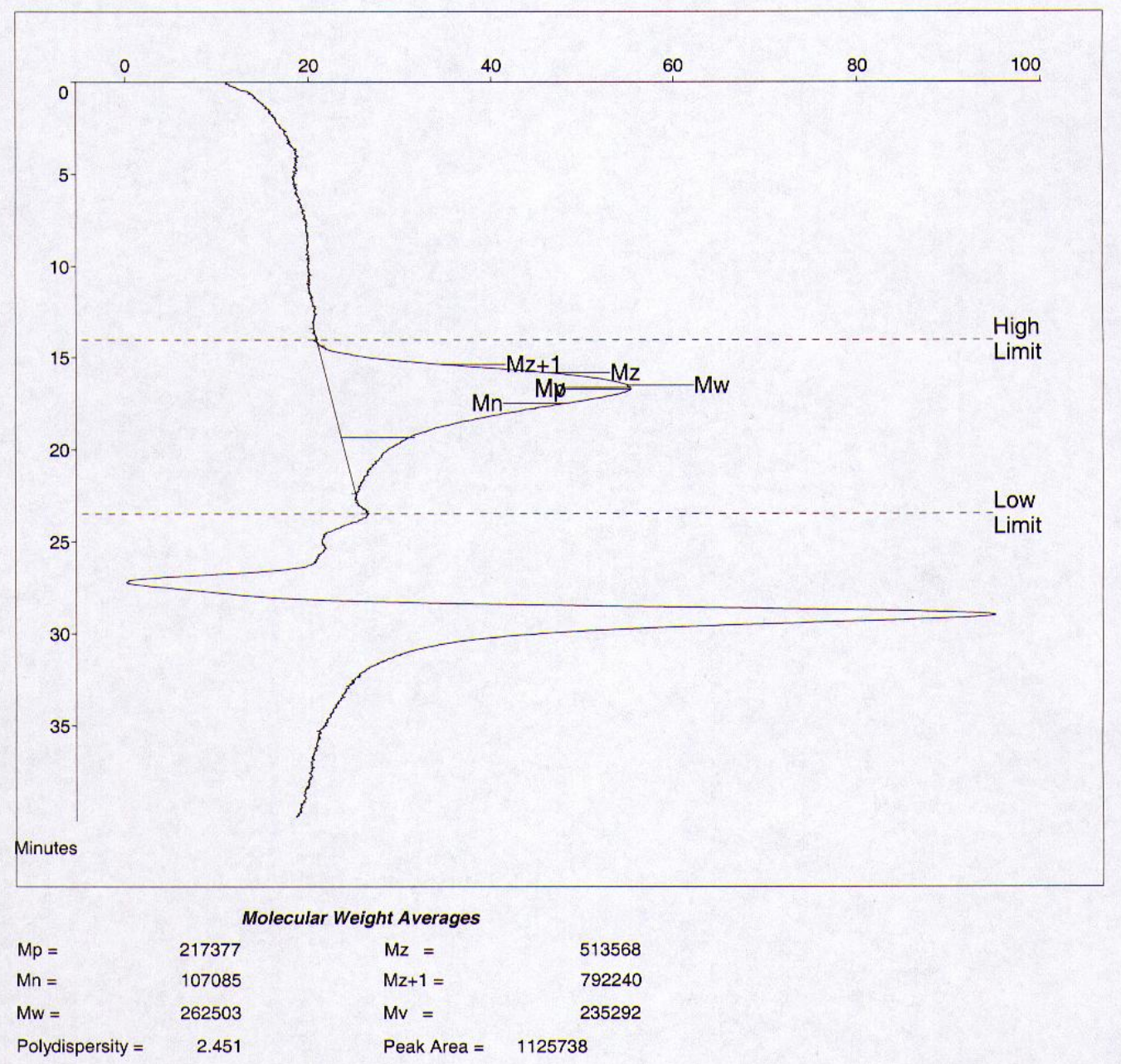


II.2. GPC of PE sample, Table 8, entry 6

Polymer Laboratories

PL Caliber GPC Software
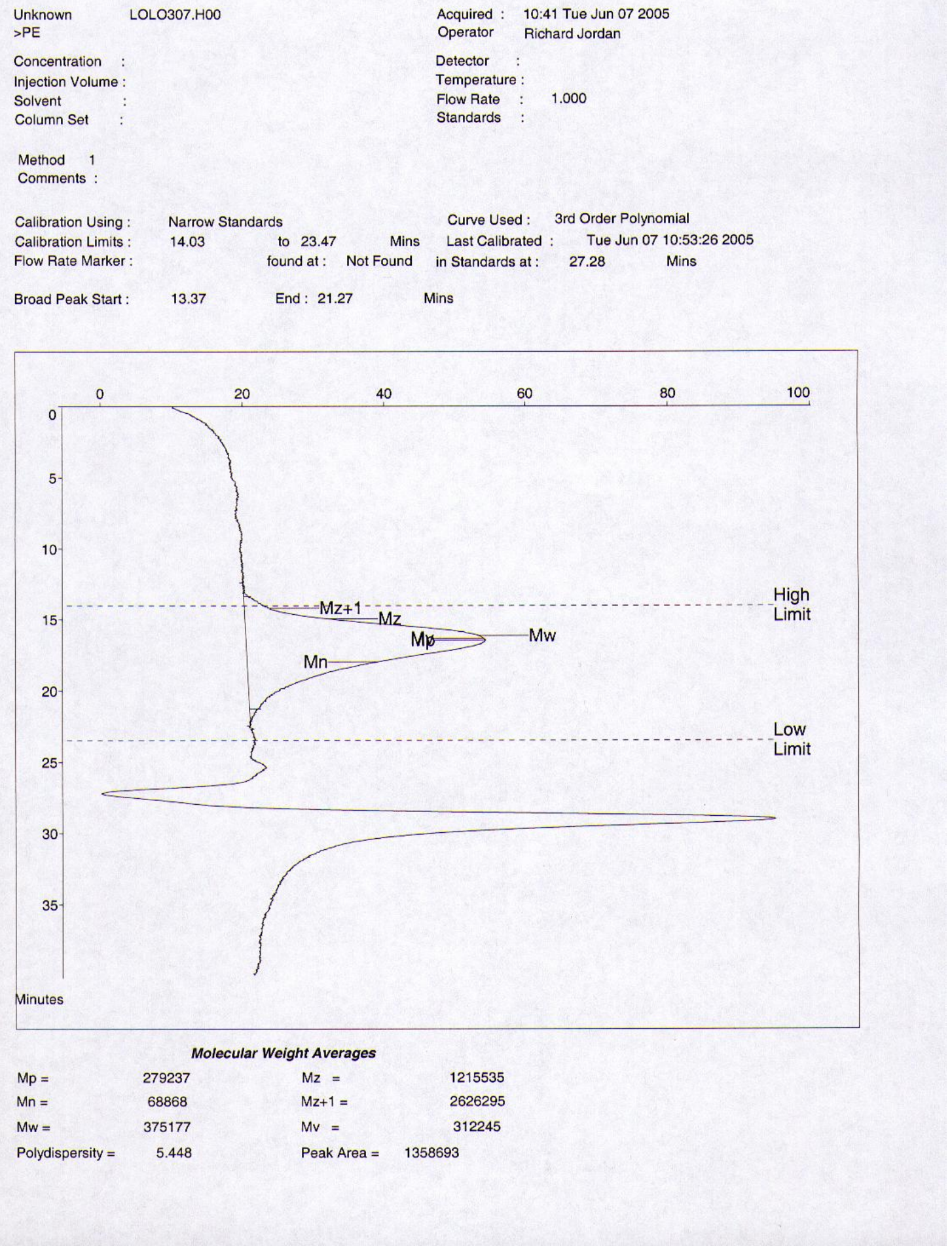
II.3. DSC of PE sample, Table 8, entry 5

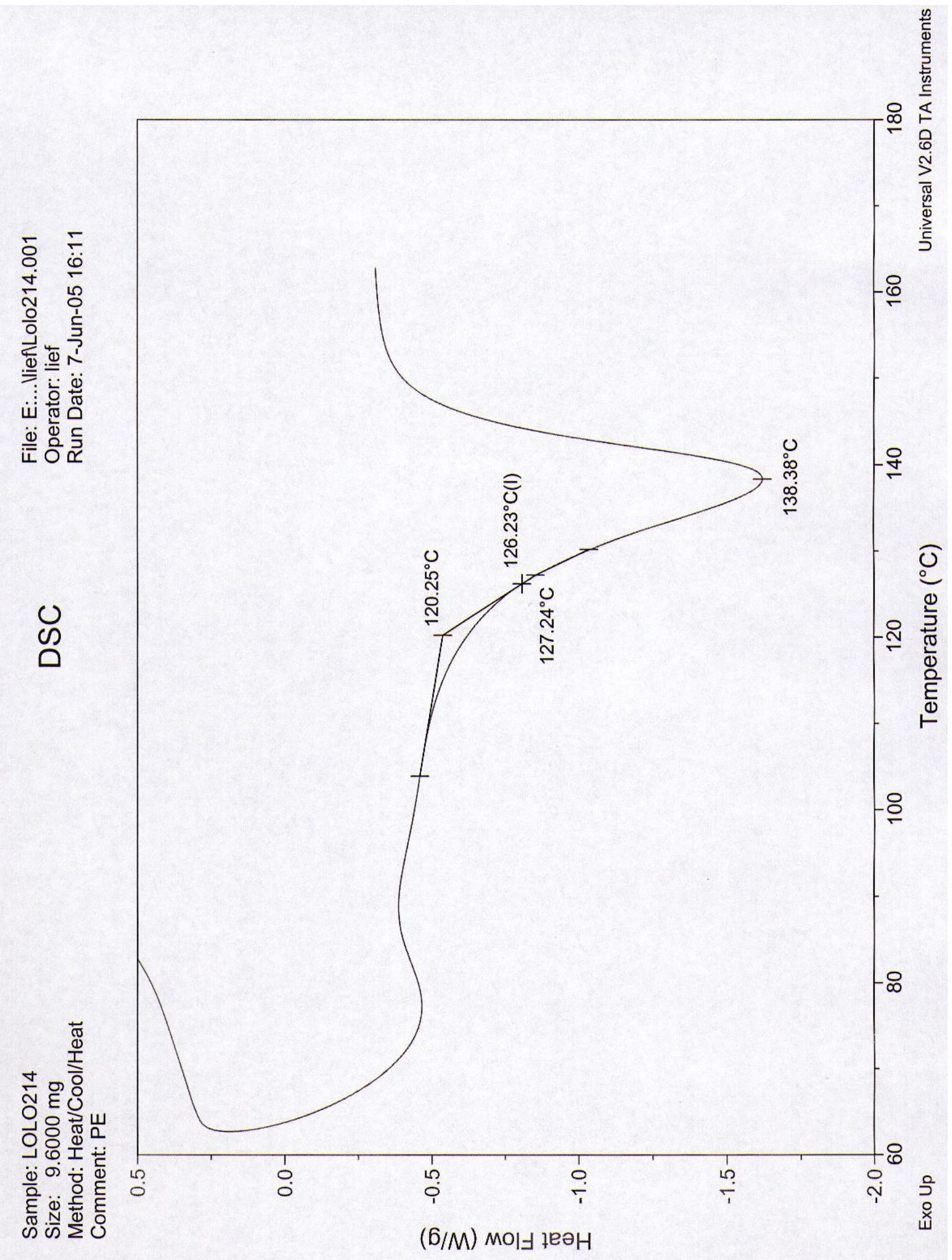


II.4. DSC of PE sample, Table 8, entry 6

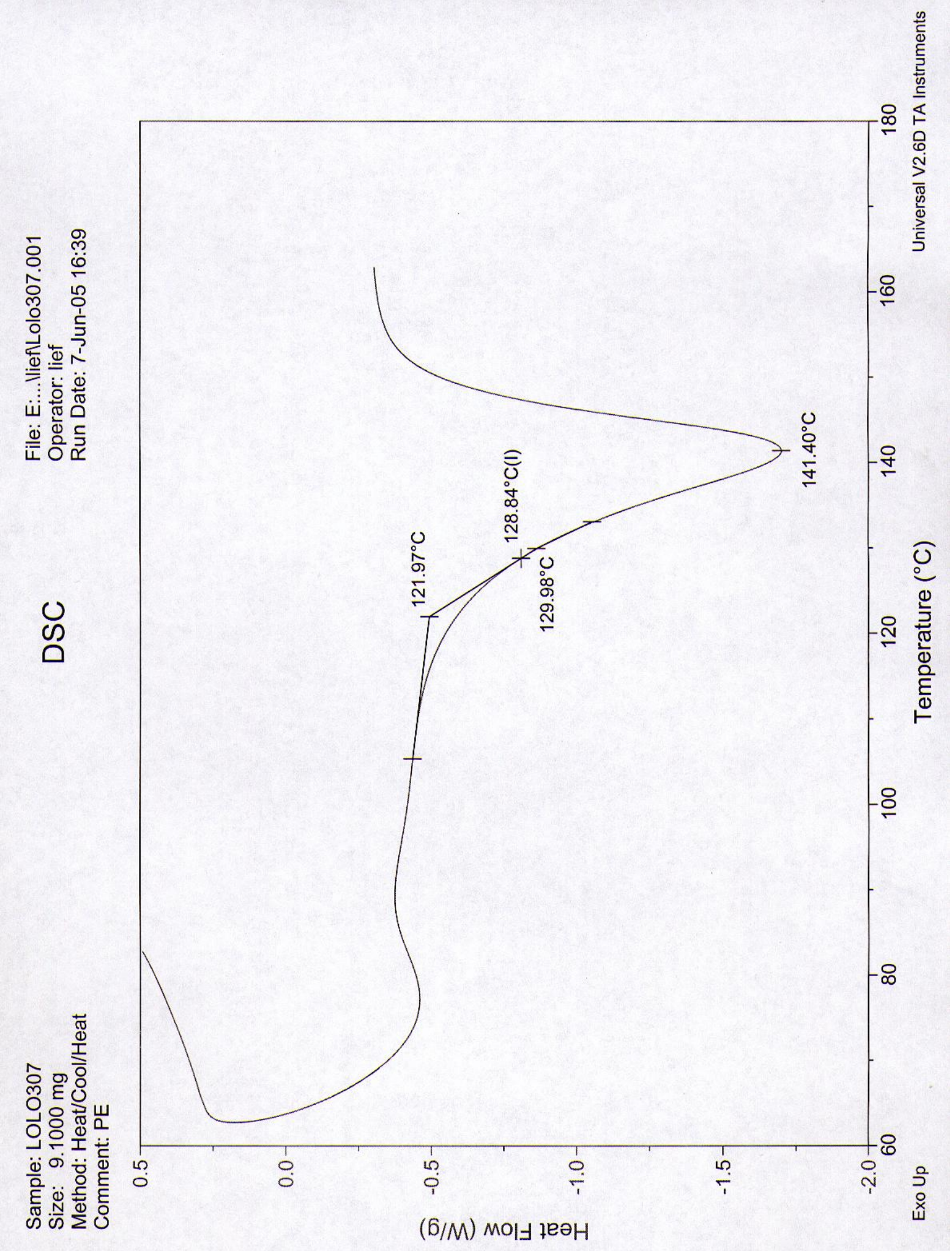

